\title{
A COPRIME ACTION VERSION OF A SOLUBILITY CRITERION OF DESKINS
}

\author{
Antonio Beltrán, Changguo Shao \\ Departamento de Matemáticas, Universidad Jaume I \\ 12071 Castellón, Spain \\ e-mail: abeltran@uji.es \\ School of Mathematical Sciences \\ University of Jinan 250022, Shandong, China \\ e-mail: shaoguozi@163.com
}

\begin{abstract}
Let $A$ and $G$ be finite groups of relatively prime orders and suppose that $A$ acts on $G$ via automorphisms. We demonstrate that if $G$ has a maximal $A$-invariant subgroup $M$ that is nilpotent and the Sylow 2subgroup of $M$ has class at most 2, then $G$ is soluble. This result extends, in the context of coprime action, a solubility criterion given by W.E. Deskins.
\end{abstract}

Keywords. soluble groups, maximal subgroups, coprime action, group action on groups.

Mathematics Subject Classification (2010): 20D20, $20 \mathrm{D} 15$.

\section{Introduction}

In [2], in the course of a study of the lattice of subinvariant subgroups in a finite group, W.E. Deskins provided an interesting solubility criterion concerning maximal subgroups: When a finite group $G$ contains a maximal subgroup $M$ that is nilpotent of class less than 3 , then $G$ is soluble. This result is similar to a theorem of B. Huppert, which originally appeared in [6], except in the case in which $M$ has a Sylow 2-subgroup of class 2. The criterion of Deskins was also in line with a theorem announced by Thompson: If a finite group $G$ has a maximal subgroup that is nilpotent of odd order, then $G$ is soluble. The crucial tool of Deskins's work, which allowed him to extend the nilpotence class to 2 instead of 1 (abelian), was the First Theorem of Grün (see for instance [5, IV.3.4]), which is an application of the transfer theory into a Sylow subgroup. Precisely, Grün's Theorem was used so as to obtain the existence of a normal complement to the maximal subgroup $M$. 
In this paper we study such results in the context in which a finite group $A$ with $(|A|,|G|)=1$ acts on $G$. We ask whether the existence of a maximal $A$-invariant subgroup in $G$ (which needs not be a maximal subgroup) satisfying the same conditions as in Deskins's theorem must imply the solubility of $G$. We give an affirmative answer.

Theorem. Let $G$ and $A$ be finite groups of coprime orders and assume that $A$ acts on $G$ by automorphisms. If $G$ has a maximal $A$-invariant subgroup that is nilpotent with a Sylow 2-subgroup of class less than 3, then $G$ is soluble.

At first sight, there seems only to be a subtle difference from Deskins's theorem, but there exists a great distinction between our development and Deskins's approach. It is not possible to use Grün's Theorem in the setting of a coprime action, and instead, we appeal to the Classification of the Finite Simple Groups. We point out that the authors have already obtained a coprime action version of the Thompson's aforementioned result [1, Theorem B]. This is not done by employing the Classification, but by transferring into the setting of coprime action results like the Glauberman-Thompson criterion for $p$-nilpotence. In fact, this result will be used in the proof of our theorem.

We denote by $\pi(G)$ the set of primes dividing the order of a group $G$. The rest of the notation is standard and all groups are supposed to be finite.

\section{Preliminaries}

We start with an elementary observation that is needed for the inductive arguments.

Lemma 2.1. Let $P$ be a finite p-group of class 2. If $A \unlhd P$, then the class of $A$ and $P / A$ is less than or equal to 2 .

We require the following theorem of Wielandt.

Theorem 2.2 (IV.7.3, [5]). Let $H$ be a Hall $\pi$-subgroup of a group $G$ which is not a Sylow subgroup of $G$. Suppose that for every $p \in \pi$ and for every Sylow $p$ subgroup $H_{p}$ of $H$, we have $\mathbf{N}_{G}\left(H_{p}\right)=H$. Then $H$ has a normal $\pi$-complement in $G$.

We also recall the Thompson subgroup. If $p$ is prime and $P$ is a $p$-group, the Thompson subgroup $\mathbf{J}(P)$ is the subgroup generated by all abelian subgroups of $P$ of maximal order. It is immediate that $\mathbf{J}(P)$ and $\mathbf{Z}(\mathbf{J}(P))$ are characteristic in $P$, and hence, these subgroups are left invariant by every automorphism acting on $P$, so in particular, by every group acting coprimely on $P$. As we said in the Introduction, in order to prove our result we need to use the celebrated Glauberman-Thompson p-nilpotence criterion.

Theorem 2.3 (Theorem 8.3.1, [4]). Let $P$ be a Sylow p-subgroup of a finite group $G$, where $p$ is an odd prime. If $\mathbf{N}_{G}(\mathbf{Z}(\mathbf{J}(P)))$ is p-nilpotent, then $G$ is p-nilpotent. 
As mentioned in the Introduction, we appeal to the Classification of the Finite Simple Groups. Precisely, we need to determine all non-abelian simple finite groups whose Sylow 2-subgroups are self-normalising as well as all those simple groups whose Sylow 2-subgroups have nilpotence class at most 2. Such groups have been classified by Kondrat'ev [7] and by Gilman and Gorenstein [3], respectively, so we can gather the list of those simple groups satisfying both conditions in the next result.

Theorem 2.4. Let $G$ be a finite non-abelian simple group and $P$ a Sylow 2subgroup of $G$. If $\mathbf{N}_{G}(P)=P$ and $P$ has class at most 2, then $G \cong \operatorname{PSL}(2, q)$, where $q \equiv 7,9(\bmod 16)$.

Proof. This is a consequence of combining the main result of [7] and Theorems 7.1 and 7.4 of [4].

We will also need to know the structure of the Sylow normalisers in $\operatorname{PSL}(2, q)$, especially for odd primes.

Lemma 2.5. Let $G=\operatorname{PSL}(2, q)$, where $q$ is a power of prime $p$ and $d=$ $(2, q+1)$. Let $r \in \pi(G)$ and $R \in \operatorname{Syl}_{r}(G)$.

(1) If $r=p$, then $\mathbf{N}_{G}(R)=R \rtimes C_{\frac{q-1}{d}}$ is a dihedral group;

(2) If $2 \neq r \mid \frac{q+1}{d}$, then $\mathbf{N}_{G}(R)=C_{\frac{q+1}{d}} \rtimes C_{2}$;

(3) If $2 \neq r \mid \frac{q-1}{d}$, then $\mathbf{N}_{G}(R)=C_{\frac{q-1}{d}} \rtimes C_{2}$;

(4) Assume $p \neq r=2$.

(4.1) If $q \equiv \pm 1(\bmod 8)$, then $\mathbf{N}_{G}(R)=R$;

(4.2) If $q \equiv \pm 3(\bmod 8)$, then $\mathbf{N}_{G}(R)=\left(C_{2} \times C_{2}\right) \rtimes C_{3}$.

Proof. This follows from [5, Theorem 2.8.27].

\section{Proof of the Theorem}

Proof. We study a minimal counter-example. Suppose then that $G$ is a minimal counter-example to the theorem and let $M$ be the nilpotent maximal $A$-invariant subgroup of $G$ with a Sylow 2-subgroup of class less than 3 . We divide the proof into the following steps.

Step 1. We can assume that $M$ is a Hall subgroup of $G$ and that $M$ does not contain any $A$-invariant normal subgroup of $G$.

If $M$ contains a non-trivial $A$-invariant normal subgroup $N$ of $G$, then by taking into account Lemma 2.1, $G / N$ satisfies the hypotheses of the theorem, so $G / N$ is soluble by minimality, and consequently, $G$ is soluble for $N$ being nilpotent. Henceforth, it can be assumed $M$ does not contain any $A$-invariant normal subgroup of $G$. 
Suppose that there exists a prime $p \in \pi(M)$ such that the Sylow $p$-subgroup of $M$ is not a Sylow $p$-subgroup of $G$. Then by elementary coprime action properties there exists an $A$-invariant Sylow $p$-subgroup $G_{p}$ of $G$ and an $A$ invariant Sylow $p$-subgroup $M_{p}$ of $M$ with $M_{p}<G_{p}$. Since $M$ is nilpotent, we have $M<\mathbf{N}_{G}\left(M_{p}\right)$. Also, $\mathbf{N}_{G}\left(M_{p}\right)$ is $A$-invariant. By the maximality of $M$ we get $\mathbf{N}_{G}\left(M_{p}\right)=G$, that is $M_{p} \unlhd G$, a contradiction with the above paragraph. This shows that $M_{p}=G_{p}$, or equivalently, $M$ is a Hall subgroup of $G$.

Step 2. We can assume that $M$ is a Sylow 2-subgroup of $G$.

Suppose that $M$ is not a Sylow subgroup of $G$. For every prime $p \in \pi(M)$ we take $P$ an $A$-invariant Sylow $p$-subgroup of $M$. Then $M \leq \mathbf{N}_{G}(P)$ and by maximality of $M$ and Step 1, it follows that $\mathbf{N}_{G}(P)=M$. Thus, we can apply Theorem 2.2, so there exists a normal complement $K$ of $M$ in $G$. Clearly, $K$ is $A$-invariant. Now let us consider the action of $M A$ on $K$. Since the orders of $M A$ and $K$ are coprime, we get that $K$ has a $M A$-invariant Sylow $q$-subgroup $Q$. Therefore $M Q \leq G$ is $A$-invariant, and by the maximality of $M$, we have $G=M Q$. However, $Q$ and $G / Q$ are soluble, so we deduce that $G$ is soluble as well, a contradiction. This shows that $M$ is a Sylow $p$-subgroup of $G$ for some prime $p$.

Next we prove that $p=2$. Assume that $p \neq 2$. Let $J=\mathbf{J}(M)$, the Thompson's subgroup of $M$, and $Z=\mathbf{Z}(J)$. Note that $Z$ and $\mathbf{N}_{G}(Z)$ are $A$ invariant by the observation made before Theorem 2.3. Since by Step $1, Z$ is not normal in $G$, we have $M \leq \mathbf{N}_{G}(Z)<G$. By the maximality of $M$, we get $M=\mathbf{N}_{G}(Z)$, so in particular it is a $p$-subgroup. Then $G$ is $p$-nilpotent by Theorem 2.3, that is, $G$ has a normal $p$-complement, say $L$, which is obviously $A$-invariant too. This means that $G=M L$ with $M \cap L=1$. The rest of the proof of this step consists in proving that $L$ is a $q$-group for some prime $q$. Indeed, take $Q$ an $A$-invariant Sylow $q$-subgroup of $L$ for some prime $q$. The Frattini argument gives $G=\mathbf{N}_{G}(Q) L$. Now, the Schur-Zassenhaus Theorem assures that $\mathbf{N}_{L}(Q)$ has complements in $\mathbf{N}_{G}(Q)$ that are conjugate in $\mathbf{N}_{G}(Q)$. Since $A$ acts on the set of complements, Glauberman's Lemma (for instance [8, Theorem 6.2.2]) implies that there exists an $A$-invariant complement $X$ of $\mathbf{N}_{L}(Q)$ in $\mathbf{N}_{G}(Q)$. As a result, $G=X \mathbf{N}_{L}(Q) L=X L$, so $X$ is an $A$-invariant complement of $L$ in $G$. Again by Glauberman's Lemma, we know that the $A$ invariant complements of $L$ are conjugate in the fixed point subgroup $\mathbf{C}_{G}(A)$, so in particular, $X=M^{c}$ for some $c \in \mathbf{C}_{G}(A)$. We conclude that $X$ is a maximal $A$-invariant subgroup of $G$. However, $X$ normalizes $Q$ and by maximality of $X$, we get $G=X Q$. This forces $L=Q$, as wanted. As a consequence, $G$ is soluble by Burnside $p^{a} q^{b}$ Theorem, a contradiction. Hence $p=2$ and $M$ is a Sylow 2-subgroup of $G$.

Step 3. We can assume that $M$ has nilpotence class 2 .

Suppose on the contrary, that the class of $M$ is not 2, so by hypothesis $M$ is abelian. As $\mathbf{N}_{G}(M)=M$ by the maximality of $M$, we have $M \leq \mathbf{Z}\left(\mathbf{N}_{G}(M)\right)$. We can apply then Burnside normal $p$-complement Theorem for $p=2$ (for 
instance [5, 2.2.6]), and we conclude that $G$ has a normal 2-complement. Now Feit-Thompson Theorem implies that $G$ is soluble, a contradiction.

Step 4. Final contradiction.

Let $N$ be a minimal $A$-invariant normal subgroup of $G$. We can assume that $N$ is not soluble; otherwise by Step $1, N$ is not contained in $M$, and by maximality we obtain $N M=G$. As a consequence, $G$ would be soluble and the proof is finished. Therefore, we can write $N=S_{1} \times \ldots \times S_{n}$ where $S_{i}$ are isomorphic non-abelian simple groups (possibly $n=1$ ). Put $S=S_{1}$, $B=\mathbf{N}_{A}(S)$ and let $T$ be a transversal of $B$ in $A$. Now, as $M$ is self-normalising in $G$ for being maximal, then $M \cap S$ is self-normalising in $S$ and it has class at most 2 by Lemma 2.1. Then by applying Theorem 2.4 , we obtain $S \cong$ $\operatorname{PSL}(2, q)$ with $q \equiv 7,9(\bmod 16)$. We distinguish separately these two cases. If $q \equiv 9(\bmod 16)$, with $q>9$, then we can certainly choose an odd prime $r \mid(q-1) / 2$ and $R$ to be a $B$-invariant Sylow $r$-subgroup of $S$. By Lemma $2.5(3)$, we know that $\left|\mathbf{N}_{S}(R)\right|=q+1$, so $\mathbf{N}_{S}(R)$ has odd index in $S$ and contains properly a Sylow 2 -subgroup of $S$. Analogously, if $q \equiv 7(\bmod 16)$, with $q>7$, there exists an odd prime $r \mid(q+1) / 2$ and we take $R$ to be a $B$-invariant Sylow $r$-subgroup of $S$. Again by Lemma 2.5(2), we know that $\left|\mathbf{N}_{S}(R)\right|=(q-1)$, so $\mathbf{N}_{S}(R)$ has odd index in $S$ and hence, it contains properly a Sylow 2-subgroup of $S$. In both cases, we put $R_{0}=\prod_{t \in T} R^{t}$, which is an $A$-invariant Sylow $r$-subgroup of $N$ because $A$ acts transitively on the $S_{i}$. We deduce that $\left|N: \mathbf{N}_{N}\left(R_{0}\right)\right|=\left|S: \mathbf{N}_{S}(R)\right|^{n}$ is odd too. Now, by the Frattini argument, $G=N \mathbf{N}_{G}\left(R_{0}\right)$ and thus, $\left|G: \mathbf{N}_{G}\left(R_{0}\right)\right|=\left|N: \mathbf{N}_{N}\left(R_{0}\right)\right|$. We conclude that $\mathbf{N}_{G}\left(R_{0}\right)$ properly contains an $A$-invariant Sylow 2-subgroup of $G$, contradicting the maximality of $M$.

Finally, suppose that $S \cong \operatorname{PSL}(2,9)$ or $\operatorname{PSL}(2,7)$. Both groups contain $\{2,3\}$-Hall subgroups, which are isomorphic to the symmetric group $S_{4}$. We remark that these subgroups are not all conjugate in $S$. If this were the case, then Glauberman's Lemma would provide an $A$-invariant Hall $\{2,3\}$-subgroup, against the maximality of $M$. But this is not the case and we give the following alternative argument. The Sylow 2-subgroups of $S$ are dihedral groups of order 8. Now, $M \cap N$ is an $A$-invariant Sylow 2-subgroup of $N$, which is the direct product of $n$ copies of such a $B$-invariant dihedral group, say $D$, of $S$. Let $K$ be the cyclic group of order 4 of $D$, which is also $B$-invariant for being characteristic, and let $K_{0}=\prod_{t \in T} K^{t}$. It is easily seen that $K_{0}$ is $A$-invariant because $A$ is acting transitively on the factors. Moreover, since $K$ is characteristic in $D$, then $K_{0}$ is characteristic in $M \cap N$, so $K_{0} \unlhd M$, that is, $M \leq \mathbf{N}_{G}\left(K_{0}\right)$. On the other hand, in both cases $S \cong \operatorname{PSL}(2,9)$ or $\operatorname{PSL}(2,7)$, we have that $K$ is normalised by an element of order 3 lying in $S$, so the same occurs with $K_{0}$ and $N$. We conclude that $\mathbf{N}_{G}\left(K_{0}\right)$ is an $A$-invariant subgroup that contains properly $M$. Again this contradicts the maximality of $M$.

\section{Acknowledgements}

The first author is partially supported by the Valencian Government, Proyecto 
PROMETEOII/2015/011 and also by Universitat Jaume I, grant P11B-201577. The second author is supported by the NNSF of China (No. 11301218) and the Nature Science Fund of Shandong Province (No. ZR2014AM020).

\section{References}

[1] Beltrán, A., Shao, C.G.: Restrictions on maximal invariant subgroups implying solvability of finite groups. Ann. Mat. Pura Appl. https://doi.org/10.1007/s10231-018-0777-1

[2] Deskins, W.E.: A condition for the solvability of a finite group. Illinois J. Math. 5, 306-313 (1961)

[3] Gilman, R., Gorenstein, D.: Finite groups with Sylow 2-subgroups of class two, I. Trans. Amer. Math. Soc. 207, 1-101 (1975)

[4] Gorenstein, D.: The Classification of finite simple groups. Simple groups and local analysis, Bull. Amer. Math. Soc. (New Series), 1(1), 43-199 (1979)

[5] Huppert, B.: Endliche Gruppen I, Springer-Verlag, Berlin-New York (1967)

[6] Huppert, B.: Normalteiler und maximale Untergruppen endlicher Gruppen, Math. Zeitschrift 60, 409-434 (1954)

[7] Kondrat'ev, A.S.: Normalizers of the Sylow 2-subgroups in finite simple groups. (Russian) Math. Zametki 78, 3, 368-376 (2005); translation in Math. Notes 78 (3-4), 338-346 (2005)

[8] Kurzweil, H., Stellmacher, B.: The Theory of Finite Groups. An introduction. Springer-Verlag, Berlin-Heidelberg-New York (2004) 